\title{
Carcinoma Ductal Invasor da Mama: Marcação Axilar Prévia ao Tratamento Neoadjuvante. Caso Clínico e Revisão da Literatura
}

\section{Invasive Ductal Carcinoma of the Breast: Axillary Marking Prior to Neoadjuvant Treatment. Case Report and Literature Review}

Charlène Viana¹, Hugo Rios¹, Nuno Barros², Luís Castro², Maria José Rocha², Arlindo Ferreira ${ }^{2}$

\section{RESUMO}

Nos doentes com cancro da mama, o tratamento neoadjuvante tem um papel fundamental na diminuição do tamanho do tumor, possibilitando uma cirurgia conservadora, sem um aumento significativo da recorrência local. Estudos mais recentes revelaram também um impacto significativo do tratamento neoadjuvante na erradicação da doença axilar. Apresentamos o caso de uma mulher de 46 anos com carcinoma ductal invasor e com doença axilar comprovada histologicamente. Após marcação de ambas as lesões com material radiopaco, a doente iniciou quimioterapia neoadjuvante seguida de cirurgia conservadora. A biópsia seletiva de gânglio sentinela revelou a ausência de células neoplásicas assim como a peça de tumorectomia, ambas marcadas previamente.

O tratamento neoadjuvante associado à marcação prévia das lesões permitiu a realização de uma cirurgia conservadora com uma resposta completa ao tratamento, para além de evitar o esvaziamento axilar.

PALAVRAS-CHAVE: Biópsia do Gânglio Sentinela; Carcinoma Ductal de Mama; Metástase Linfática; Neoplasias da Mama; Terapia Neoadjuvante 


\begin{abstract}
In patients with breast cancer, neoadjuvant treatment plays a fundamental role in decreasing tumor size allowing conservative surgery without a significant increase in local recurrence. More recent studies have also revealed a significant impact of neoadjuvant treatment on eradication of axillary disease.

This is the case of a 46-year-old female with invasive ductal carcinoma and with axillary disease proven histologically. After marking both lesions with radiopaque material, the patient started neoadjuvant chemotherapy followed by conservative surgery. Selective sentinel lymph node biopsy revealed the absence of neoplastic cells as well as piece of tumorectomy, both previously marked.

Neoadjuvant treatment associated with previous marking lesions, allowed conservative surgery with a complete response to treatment, besides avoiding axillary dissection.

KEYWORDS: Breast Neoplasms; Carcinoma, Ductal, Breast; Lymphatic Metastasis; Neoadjuvant Therapy; Sentinel Lymph Node Biopsy
\end{abstract}

\section{INTRODUÇÃO}

O cancro da mama é a neoplasia mais frequente do sexo feminino, afetando uma em cada nove mulheres. Constitui a causa mais frequente de mortalidade na faixa etária entre os 35 e os 55 anos de idade na União Europeia. ${ }^{1}$

De forma genérica, em termos histológicos o cancro da mama pode ser dividido em não invasor (carcinoma ductal in situ e carcinoma lobular in situ) ou invasor (carcinoma ductal invasor - CDI, carcinoma lobular invasor, doença de Paget e carcinoma inflamatório).2,3 Os carcinomas invasores da mama constituem um grupo heterogéneo de lesões, sendo o CDI o mais comum. ${ }^{4} \mathrm{O}$ principal objetivo na identificação dos variados tipos de carcinomas invasores da mama é permitir a previsibilidade do comportamento do tumor e a resposta ao tratamento. 3,4

A escolha do tratamento tem em conta vários fatores: status hormono-recetor (HR) e HER-2, status de menopausa, subtipo molecular do tumor, realização de tratamentos prévios e toxicidade associada, tempo de sobrevida sem doença, carga tumoral (número e locais de doença metastática), idade, "performance status", comorbilidades, necessidade premente de controlo da doença/sintomas, fatores psicológicos e socioeconómicos, tratamentos existentes no país em que se encontra o doente e a vontade do mesmo. ${ }^{5}$

O tratamento neoadjuvante era aplicado, no passado, a doentes com doença inoperável, todavia, nas últimas décadas, este tratamento mostrou-se benéfico em doentes com estádios mais precoces da doença. Essa mudança deu-se ao facto de os estudos revelarem que, quando aplicado, o tratamento neoadjuvante permite diminuir o tamanho do tumor, possibilitando uma cirurgia conservadora em doentes selecionados, sem um aumento significativo da recorrência local. ${ }^{6-8}$ Em 2001, num estudo da European Organisation for Research and Treatment of Cancer (EORTC) verificou-se que 23\% dos doentes com indicação prévia para mastectomia foram submetidos a cirurgia conservadora após tratamento neoadjuvante. $^{8}$

A existência de resposta patológica completa (pCR) é um fenómeno descrito em muitos estudos e pressupõe a ausência de tumor invasivo residual na avaliação histológica da peça cirúrgica após o tratamento neoadjuvante. A existência de pCR tem sido usada como fator de prognóstico importante em muitos estudos, uma vez que aqueles que respondem parcialmente parecem ter uma menor taxa de sobrevida do que aqueles que apresentam pCR. ${ }^{6}$ Para além da diminuição do tamanho do tumor, o tratamento neoadjuvante permite uma diminuição da doença axilar na ordem dos 23\%, convertendo gânglios clinicamente positivos em gânglios patologicamente negativos. Doentes que adquiriram pCR no tumor primário têm maior probabilidade de ter doença axilar negativa, o que é de extrema importância uma vez que o nível de envolvimento axilar após quimioterapia (QT) é um fator preditivo importantíssimo de resposta ao tratamento. ${ }^{7}$ A taxa de pCR é variável na literatura, apresentando valores entre 4\% e 29\%. ${ }^{9}$

A análise do subtipo molecular do tumor (luminal A/B, basal, HER2+ e normal-like) revelou tratar-se de extrema importância dada a variabilidade de comportamentos e respostas ao tratamento neoadjuvante. Este conhecimento é fundamental para uma melhor seleção do tratamento adequado a cada doente. Doentes com subtipos já conhecidos como sendo mais agressivos são submetidos a terapias específicas mais eficazes; por outro lado, a identificação de subtipos que não irão responder à terapêutica permite limitar a toxicidade e optar por tratamentos alternativos numa fase mais precoce. Como exemplo disso temos o trastuzumab e outros fármacos 
anti-HER2 (ex.: pertuzumab, lapatinib, T-DM) que vieram revolucionar o tratamento do cancro HER2+.6,10

O tratamento neoadjuvante, associando terapias hormonais em mulheres pós-menopáusicas com tumores $\mathrm{HR}+$, permitiu diminuir o tamanho do tumor e a realização posterior de cirurgias conservadoras. Os inibidores da aromatase parecem ser superiores ao tamoxifeno com melhor resposta e melhores perfis de toxicidade. ${ }^{6}$

Apresentamos o caso de uma mulher com carcinoma ductal invasor, cuja terapêutica neoadjuvante permitiu a realização de uma cirurgia conservadora sem necessidade de esvaziamento axilar.

\section{CASO CLÍNICO}

Apresentamos o caso de uma mulher de 46 anos, casada, com menarca aos 11 anos e ciclos regulares. Como método contracetivo recorria ao anticoncetivo oral. Tratava-se de uma mulher com duas gestações e dois partos (IIG/IIP).

Segundo a doente, detetou em autoexame, um nódulo na mama direita. Ao exame objetivo comprovou-se uma nodularidade no quadrante supero-externo (QSE) da mama direita com cerca de 3,5 cm associada a retração da pele suprajacente assim como uma adenopatia com $2 \mathrm{~cm}$ no cavado axilar direito. Trazia consigo uma mamografia e ecografia recentes que comprovavam a existência de uma modularidade de $\sim 3 \mathrm{~cm}$ no QSE da mama direita (Fig. 1).

Já na nossa instituição, na Unidade de Senologia, a doente foi submetida a microbiópsias ecoguiadas, quer da lesão, quer da adenopatia suspeita. As microbiópsias da lesão da mama revelaram: "CDI GIII; recetores de estrogénio (ER) 90\% positivo; recetores de progesterona (PR) 0\% negativo; Her2/neu 1+ (negativo); Ki 67\% - 78\%". As microbiópsias da adenopatia axilar confirmaram tratar-se de metástase ganglionar da neoplasia previamente descrita (Fig. 2). Tratava-se, portanto, de um CDI estádio IIB (T2N1). Após a confirmação histológica das lesões, procedemos à marcação das mesmas com marcadores radiopacos (clipes de titânio) (Fig. 3). Os restantes meios complementares de estadiamento realizados (análises, tomografia computorizada toraco-abdomino-pélvica e cintilograma ósseo) não mostraram evidência de lesões à distância.

Procedemos à discussão do caso clínico em Consulta de Grupo Multidisciplinar (CGM) de Senologia, envolvendo médicos de Senologia, Oncologia, Anatomia Patológica, Radiologia e Radioterapia, e a decisão foi a de iniciar tratamento com QT neoadjuvante com adriamicina, ciclo- fosfamida - docetaxel (AC-D). Os tratamentos decorreram sem intercorrências de relevo, e após os seis ciclos de QT realizamos novo reestadiamento da doença que revelou resposta clínica e radiológica completas (Fig. 4). Em nova discussão do caso clínico em CGM, a decisão foi a do tratamento cirúrgico.

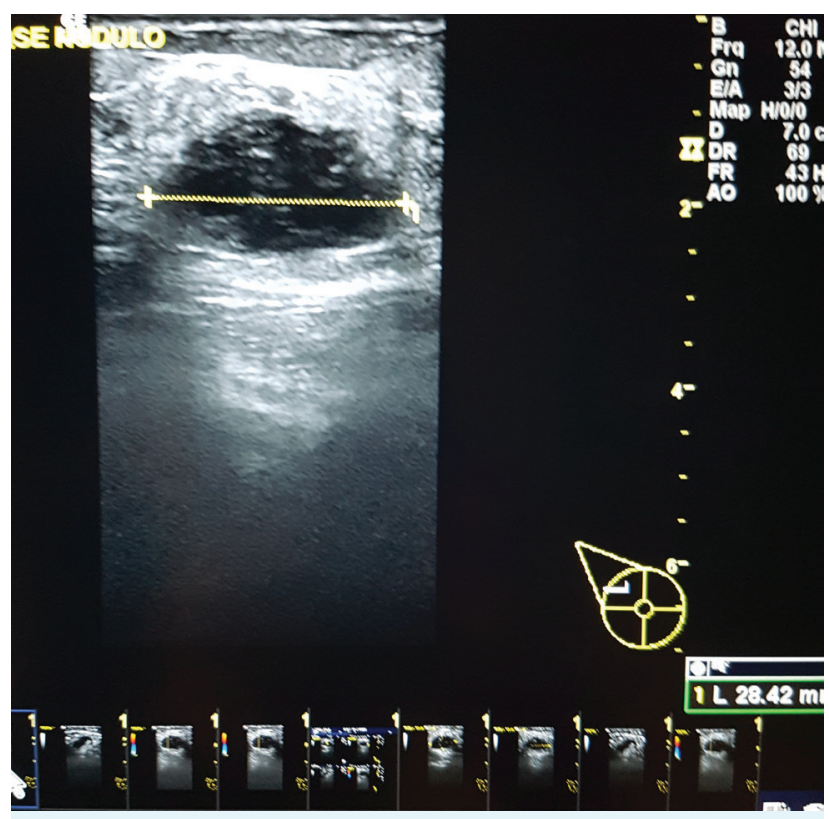

FIGURA 1. Ecografia mamária - nódulo na mama direita com 28,4 mm de maior diâmetro.

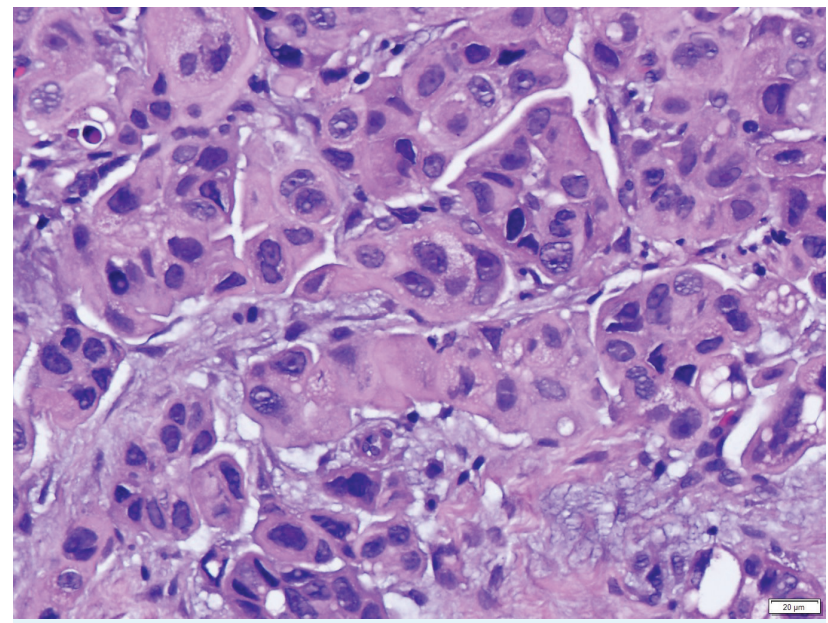

FIGURA 2. Biópsia de nódulo da mama mostrando carcinoma ductal invasor grau III.

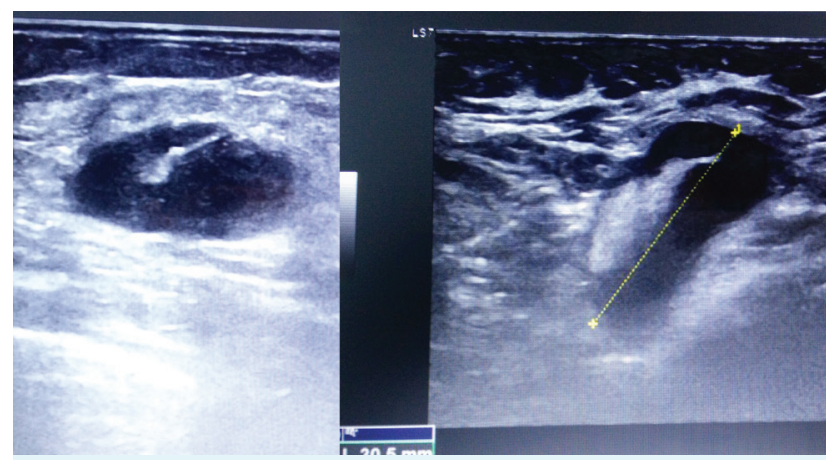

FIGURA 3. Imagens ecográficas de marcação com clipes. À esquerda, marcação da neoplasia. À direita, marcação de adenopatia com 30,5 mm de maior diâmetro. 


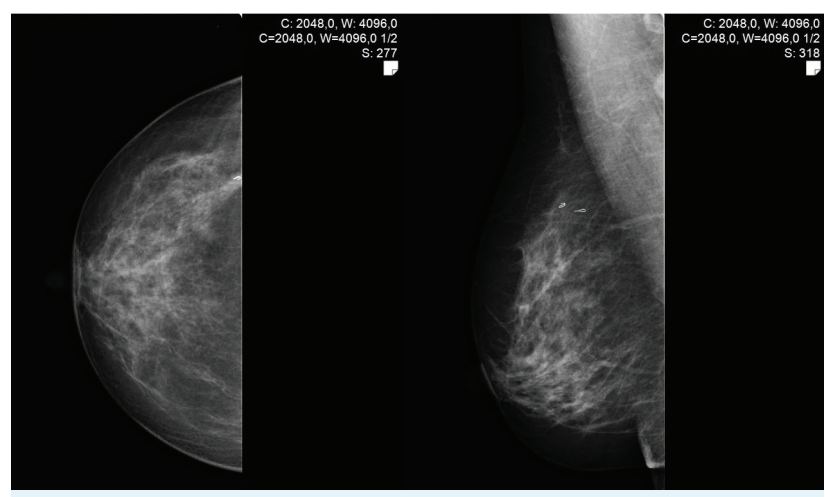

FIGURA 4. Mamografia de reestadiamento, sem evidência de neoplasia. Vizualizam-se os clipes de anterior marcação.

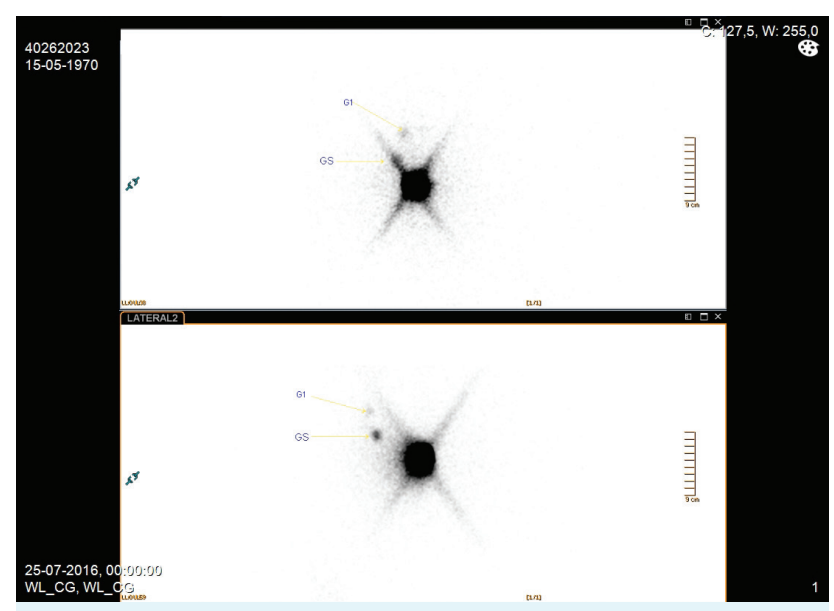

FIGURA 5. Linfocintigrafia com Tc 99 m para marcação de gânglio sentinela.

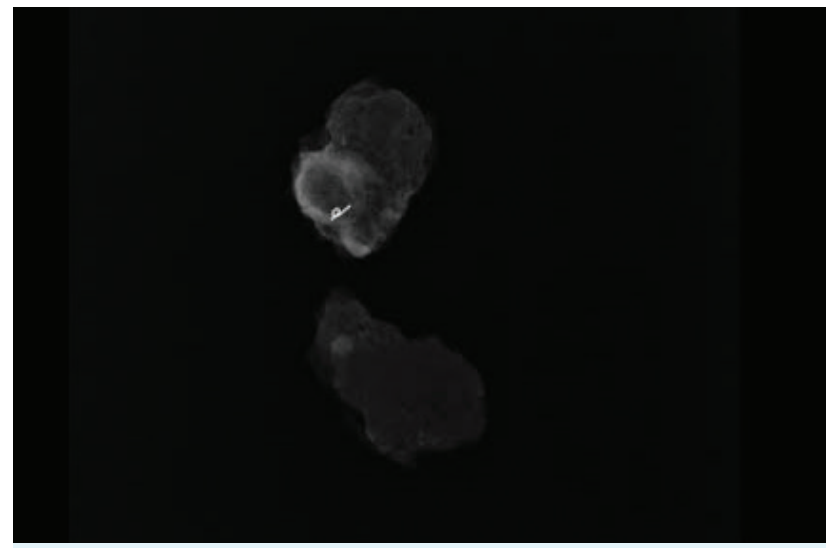

FIGURA 6. Radiografia de duas adenopatias referenciadas como gânglio sentinela, a de cima marcada com clipe.

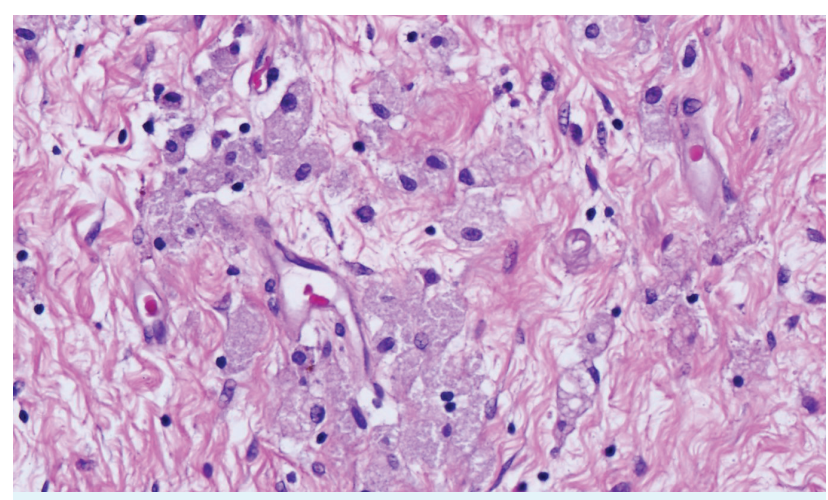

FIGURA 7. Resultado histológico definitivo da peça cirúrgica sem evidência de neoplasia. Verificada extensa fibrose e infiltrado inflamatório linfoplasmocitário com histiócitos.
Quatro semanas após o término da QT neoadjuvante, a doente foi submetida a tumorectomia guiada por arpão e biópsia seletiva de gânglio sentinela (BSGS), englobando o nódulo previamente marcado. O gânglio sentinela é identificado através da marcação prévia por linfocintigrafia com tecnécio 99 m (Fig. 5) e com azul patente infiltrado na região periareolar (no bloco, imediatamente antes da cirurgia). Durante a cirurgia, procedemos à confirmação radiológica da existência do clipe numa das adenopatias removidas (Fig. 6). Realizamos exame extemporâneo de ambas as peças que revelou: "Dois gânglios linfáticos, um deles com clipe (...) não se observando células epiteliais malignas; Tumorectomia (...) sem nódulo bem definido (...)". A cirurgia decorreu sem intercorrências e a doente teve alta 48 horas após a cirurgia.

O pós-operatório decorreu sem complicações com boa evolução cicatricial. O resultado histológico definitivo confirmou a ausência de células malignas quer nas adenopatias quer na peça de tumorectomia enviadas, revelando apenas alterações cicatriciais em ambas as peças compatíveis com tecido neoplásico prévio (ypTO NO(sn) CR) (Fig. 7).

Numa terceira discussão em CGM a decisão foi a de tratamento adjuvante com radioterapia (RT) e hormonoterapia (HT) com tamoxifeno. Vinte e um meses após o início dos tratamentos e após término das sessões de RT a doente encontra-se bem, sem queixas de relevo e sem evidência de recidiva clínica ou radiológica.

\section{DISCUSSÃO}

A maioria dos carcinomas invasores da mama são adenocarcinomas e a sua classificação histopatológica tem como base padrões de crescimento e características citológicas. ${ }^{3,4} \mathrm{O} \mathrm{CDI}$ é o mais comum, correspondendo a 70\% - 75\% dos casos. Macroscopicamente pode ter de poucos milímetros a vários centímetros de tamanho. Microscopicamente, mais de 50\% não apresenta nenhum padrão específico, 70\% a 80\% apresenta positividade para ER e 15\% a 30\% é positivo para o HER2. ${ }^{4}$

A tomada de decisões relativamente à orientação e tratamento do cancro da mama é complexa e deve incluir especialistas de várias áreas em reunião multidisciplinar. Dessas decisões devem fazer parte também o doente (e a família se o doente assim o pretender), para que este seja incluído na consciencialização e tomada de decisões em relação às várias opções terapêuticas. ${ }^{5}$

Vários são os fatores de prognóstico no cancro da mama invasor que, após identificados, vão permitir prever o comportamento do tumor ao longo do tempo, assim 
como a resposta ao tratamento.,4 Os principais fatores de prognóstico determinantes na resposta terapêutica são: o estádio linfático axilar (o mais importante dos fatores de prognóstico uma vez que a sobrevida livre de doença diminui quanto maior o número de gânglios linfáticos atingidos) e o tamanho do tumor. Outros fatores de prognóstico são: tipo e grau histológico; status de invasão linfovascular; status dos recetores hormonais (ex. os tumores hormono-dependentes, nomeadamente os luminal A, têm melhor prognóstico e associam-se a baixa taxa de recidiva e a maior sobrevida) ${ }^{4,11}$; status HER2 (quando positivo significa uma melhor resposta à QT para além de ter indicação para terapia molecular). ${ }^{4}$

Quando indicada, uma estratégia multidisciplinar com tratamento sistémico inicial pode permitir uma cirurgia conservadora para além de fornecer informações relativamente à biologia do tumor e à resposta do doente aos tratamentos. ${ }^{8} \mathrm{~A}$ QT neoadjuvante tem indicação nos casos de doença localmente avançada, contudo, o benefício em estádios mais precoces tem sido crescente, nomeadamente em casos borderline em que a relação entre a neoplasia e a mama livre de doença não permitiria realizar uma cirurgia conservadora com resultados estéticos aceitáveis., ${ }^{4,12}$

No caso de se optar pela cirurgia conservadora, para além do objetivo fundamental de pretender a excisão completa do tumor com margens livres, o resultado estético ganha relevante importância. A tumorectomia, aplicável em tumores estádio I/II, pode ser considerada em casos selecionados como sendo: tumores com menos de $4 \mathrm{~cm}$ e em que há uma relação favorável entre o tamanho do tumor e da mama livre de doença; não aderência aos planos profundos nem envolvimento da pele pelo tumor; ausência de multicentricidade (pode ser multifocal); ausência de suspeita de lesão ganglionar axilar²; ausência de histologia de carcinoma lobular. ${ }^{6}$

Doentes cujo tratamento inicial é a terapêutica neoadjuvante, devem ter sempre uma caracterização clínica e radiológica da axila. No caso de haver gânglios linfáticos suspeitos, é recomendada a realização de uma biópsia percutânea à região axilar. No passado, perante envolvimento ganglionar aquando do diagnóstico, o esvaziamento ganglionar (níveis I/II) era realizado aquando da cirurgia, independentemente da resposta à QT neoadjuvante. ${ }^{4,6}$ Recentemente, estudos revelaram que, para além de permitir a cirurgia conservadora da mama, o tratamento neoadjuvante tem também impacto na erradicação da doença axilar. A BSGS (mesmo que clinicamente negativo) após término do tratamento neoadjuvante, parece ser segura e eficaz, permitindo diminuir grandemente as taxas de morbilidade associadas ao esvazia- mento axilar. No caso dos gânglios positivos previamente ao tratamento neoadjuvante, o papel da BSG é mais controverso. 6 O estudo ACOZOG Z1071 revelou que, em doentes submetidos a tratamento neoadjuvante, a BSGS após o tratamento teve 12,9\% de falsos negativos, contudo, os autores dizem que esta taxa pode ser meIhorada com a marcação prévia do gânglio com material radiopaco e com a exérese de pelo menos dois gânglios. Nestes casos, após a marcação prévia ao tratamento neoadjuvante do gânglio suspeito, a taxa de falsos negativos desceu para 7,4\%.4,6,13 No caso da nossa doente procedemos à marcação da neoplasia e da adenopatia. Com este procedimento, para além de identificarmos o local prévio da neoplasia, conseguimos comprovar que a adenopatia excisada e referenciada como gânglio sentinela correspondia à adenopatia previamente metastática. A evidência, em exame extemporâneo, de ausência de células neoplásicas (comprovada posteriormente na peça cirúrgica), permitiu-nos evitar, com segurança, o esvaziamento ganglionar axilar e toda a morbilidade associada ao mesmo.

A resposta à neoadjuvância pressupõe três níveis de resposta: clínica, radiológica e histológica. ${ }^{4}$ A principal preocupação aquando da realização da cirurgia conservadora é a recidiva local. Alguns estudos revelaram um aumento de $22 \%$ do risco de recidiva locorregional após terapia neoadjuvante nos casos em que, após resposta clínica e radiológica completa, as doentes não foram submetidas a cirurgia (apenas a RT), o que revela que a resposta completa não é acompanhada de ausência completa de doença, motivo pelo qual a cirurgia nunca deve ser eliminada. ${ }^{14,15}$ A cirurgia deve ser realizada, idealmente, quatro semanas após o fim do tratamento. A área inicialmente atingida pelo tumor não necessita obrigatoriamente de ser removida na totalidade desde que a área previamente atingida esteja marcada com material radiopaco. ${ }^{4}$

A HT neoadjuvante é opção primária nos tumores com positividade para ER e PR. Vários estudos demonstraram que os tumores ER+ têm uma baixa taxa de resposta à QT neoadjuvante quando comparados com outros subtipos. Após o desenvolvimento das terapias hormonais e a sua inclusão no tratamento neoadjuvante, verificaram-se respostas clínicas importantes com diminuição do tamanho do tumor e a realização de cirurgias conservadoras, apesar da baixa taxa de respostas completas. Os doentes com melhor resposta à HT neoadjuvante são as mulheres pós-menopáusicas com tumores grandes ou localmente avançados ER+, mulheres mais idosas que tolerariam mal uma QT mais agressiva ou aquelas que não são candidatas a cirurgia dadas as 
múltiplas comorbilidades. Vários estudos compararam a eficácia do tamoxifeno (inibidor competitivo do estradiol) com os inibidores da aromatase (Ex.: anastrozol, letrozol, exemestano) e todos mostraram a superioridade destes últimos como terapêutica hormonal neoadjuvante nas mulheres pós-menopáusicas com diminuições importantes do tamanho do tumor. 6,16 No caso específico do cancro luminal avançado, os avanços recentes mais importantes são, sem dúvida, a introdução dos inibidores da CDK4/6 como sendo o palbociclib que, quando combinado com os inibidores da aromatase, parecem ter vantagem como primeira linha (fase II do estudo PALOMA1) com boas taxas de resposta e baixa toxicidade. $^{5}$

A neoadjuvância com QT associada à terapêutica anti-HER2, tem indicação nos casos HER2+, com elevada taxa de resposta completa. ${ }^{4}$ Exemplo desses fármacos é o trastuzumab que veio revolucionar o tratamento do cancro HER2+ com aumento das taxas de resposta completa e sobrevida quando associado a QT.5,17 Em contrapartida, estudos mais recentes revelaram taxas de resposta completa de $16 \%$ com o uso do trastuzumab sem QT, o que mostra que alguns doentes poderão beneficiar do tratamento com anti-HER2 sem QT e, portanto, com menos efeitos adversos. ${ }^{6}$

O cancro da mama triplo negativo é distinguido pela falta de expressão de recetores de estrogénio, progesterona e HER2 e é, caracteristicamente, de mau prognóstico. ${ }^{6}$ A falta de recetores no tumor triplo negativo dificulta o desenvolvimento de fármacos alternativos. Dada a semelhança desta doença com os cancros associados ao BRCA1, o uso de drogas contendo platina (atuação diretamente no DNA) parece ter particular interesse. ${ }^{18}$ Nos últimos dois anos, o estudo TNT comparando o docetaxel e a carboplatina em doentes com tumores triplo negativos veio demonstrar superioridade da carboplatina nos doentes BRCA+, enquanto que nos BRCA negativos não pareceu haver diferenças entre os dois fármacos. Mais estudos são necessários para uma caracterização biológica destes tumores de forma a criar novas terapêuticas mais dirigidas e eficazes. ${ }^{5}$

Relativamente ao tratamento adjuvante, alguns estudos recomendam o tratamento em doentes com gânglios positivos e nos tumores com mais de $1 \mathrm{~cm}$, independentemente das outras características do tumor. A RT tem um papel fundamental no tratamento do cancro da mama através da erradicação da doença subclínica após o tratamento cirúrgico. Reduz a recorrência local e aumenta a sobrevida das doentes com cancro da mama submetidas a cirurgia conservadora assim como nas doentes submetidas a mastectomia com gânglios posi- tivos. A QT adjuvante deve ser considerada nos doentes com: recetores hormonais negativos, doença HER2+, doença axilar positiva, doentes hormonodependentes com tumores com mais de $2 \mathrm{~cm}$, tumores GII/GII atendendo à idade, entre outros. Nos doentes sem doença axilar há testes moleculares que, consoante o resultado, permitem poupar a QT a um subgrupo significativo de doentes. ${ }^{2,5}$ A HT deve ser considerada nos doentes com $\mathrm{HR}+$ e com doença metastática indolente. Atualmente, o tamoxifeno é o tratamento de escolha para doentes pré-menopáusicas e os inibidores da aromatase (ex. anastrozol) para mulheres pós-menopáusicas. ${ }^{2}$

No caso apresentado, a doente teve resposta completa ao tratamento, comprovada clínica, imagiológica e histologicamente. Dada a realização de cirurgia conservadora e a existência de ER+, realizou RT e HT adjuvantes. Este é um caso de sucesso em que, após a marcação prévia das lesões e do tratamento neoadjuvante, foi possível a realização de uma cirurgia conservadora sem esvaziamento axilar, e com regressão locorregional completa comprovada.

Mais estudos são necessários para que seja possível responder a questões fundamentais relativas ao cancro da mama. Exemplos dessas questões são a própria biologia dos tumores, toda a heterogeneidade associada e os mecanismos de resistência às terapêuticas desenvolvidas. Só com esse conhecimento será possível, no futuro, usufruir das novas tecnologias (ex.: sequência genética e sistemas biológicos) para criar avanços nessas matérias. $^{5}$

CONFLITOS DE INTERESSE: Os autores declaram não ter qualquer conflito de interesse na realização do presente trabalho.

FONTES DE FINANCIAMENTO: Não houve qualquer fonte de financiamento na realização do presente trabalho.

CONFIDENCIALIDADE DOS DADOS: Os autores declaram ter seguido os protocolos da sua instituição acerca da publicação dos dados de doentes.

PROTEÇÃO DE PESSOAS E ANIMAIS: Os autores declaram que os procedimentos seguidos na elaboração do presente trabalho estão em conformidade com as normas das comissões de investigação clínica e de ética, bem como da declaração de Helsínquia e da Associação Médica Mundial.

CONFLICTS OF INTEREST: The authors declare that they have no conflicts of interest.

FINANCIAL SUPPORT: This work has not received any contribution, grant or scholarship. 
CONFIDENTIALITY OF DATA: The authors declare that they have followed the protocols of their work center on the publication of data from patients.

PROTECTION OF HUMAN AND ANIMAL SUBJECTS: The authors declare that the procedures followed were in accordance with the regulations of the relevant clinical research ethics committee and with those of the Code of Ethics of the World Medical Association (Declaration of Helsinki).

\section{REFERÊNCIAS}

1. Direção-Geral da Saúde. Norma Nº 051/2011 de 27/12/2011: Abordagem Imagiológica da Mama Feminina. Lisboa: DGS;2011.

2. Sthehr W, Reid M. Surgical Handbook. $6^{\text {th }}$ ed. Amsterdam: Elsevier; 2008.

3. Barroso-Sousa R, Metzger-Filho O. Differences between invasive lobular and invasive ductal carcinoma of the breast: results and therapeutic implications. Ther Adv Med Oncol. 2016;8:261-6.

4. Fuhrman GM, King TA. Multidisciplinary breast management. Surg Clin North Am. 2013;93:xvii-xviii.

5. Cardoso F, Costa A, Norton L, Senkus E, Aapro M, André F, et al. ESO-ESMO 2nd international consensus guidelines for advanced breast cancer (ABC2). Ann Oncol. 2014;25:1871-88.

6. Teshome M, Hunt KK. Neoadjuvant therapy in the treatment of breast cancer. Surg Oncol Clin N Am. 2014;23:505-23.

7. Fisher B, Bryant J, Wolmark N, Mamounas E, Brown A, Fisher ER, et al. Effect of preoperative chemotherapy on the outcome of women with operable breast cancer. J Clin Oncol. 1998;16:2672-85.

8. Van der Hage JA, van de Velde CJ, Julien JP, Tubiana-Hulin M, Vandervelden C, Duchateau L. Preoperative chemotherapy in primary operable breast cancer: results from the European Organization for Research and Treatment of Cancer trial 10902. J Clin Oncol. 2001;19:4224-37.

9. Mauri D, Pavlidis N, loannidis JP. Neoadjuvant versus adjuvant systemic treatment in breast cancer: a meta-analysis. J Natl Cancer Inst. 2005;97:188-94.

10. Perou CM, Sorlie T, Eisen MB, van de Rijn M, Jeffrey SS, Rees CA, et al. Molecular portraits of human breast tumours. Nature. 2000;406:747-52.

11. Kuehn T, Bauerfeind I, Fehm T, Fleige B, Hausschild M, Helms $\mathrm{G}$, et al. Sentinel-lymph-node biopsy in patients with breast cancer before and after neoadjuvant chemotherapy (SENTINA): a prospective, multicentre cohort study. Lancet Oncol. 2013;14:609-18.

12. Bobin JY, Al-Khaledi K, Ahmad J. Breast conservative surgery for operable invasive ductal carcinoma after neoadjuvant chemotherapy or hormonal therapy- a challenge for breast surgeon: a review based on literature and experience. Gulf J Oncolog. 2011;9:45-51.

13. Boughey JC, Suman VJ, Mittendorf EA, Ahrendt GM, Wilke LG, Taback B, et al. Sentinel Lymph Node Surgery after Neoadjuvant Chemotherapy in Patients with Node-Positive Breast Cancer: The American College of Surgeons Oncology Group (ACOSOG) Z1071 Clinical Trial. JAMA. 2013;310:1455-61.

14. Mauri D, Pavlidis N, Ioannidis JP. Neoadjuvant versus adjuvant systemic treatment in breast cancer: a meta-analysis. J Natl Cancer Inst. 2005;97:188-94.
15. Weksberg DC, Allen PK, Hoffman KE, Litton JK, Strom EA, Shah RR, et al. Outcomes and predictive factors for salvage therapy after local-regional recurrence following neoadjuvant chemotherapy and breast conserving therapy. Ann Surg Oncol. 2013;20:3430-7.

16. Eiermann W, Paepke S, Appfelstaedt J, Llombart-Cussac A, Eremin J, Vinholes J, et al. Preoperative treatment of postmenopausal breast cancer patients with letrozole: A randomized double-blind multicenter study. Ann Oncol. 2001;12:1527-32.

17. Buzdar AU, Ibrahim NK, Francis D, Booser DJ, Thomas ES, Theriault RL, et al. Significantly higher pathologic complete remission rate after neoadjuvant therapy with trastuzumab, paclitaxel, and epirubicin chemotherapy: results of a randomized trial in human epidermal growth factor receptor 2-positive operable breast cancer. J Clin Oncol. 2005;23:3676-85.

18. Chang HR, Glaspy J, Allison MA, Kass FC, Elashoff R, Chung DU, et al. Differential response of triple-negative breast cancer to a docetaxel and carboplatin-based neoadjuvant treatment. Cancer. 2010;116:4227-37. 